\title{
Littérature et démocratie
}

\section{Alexandre GEFEN, CNRS}

Le réengagement des écrivains et la repolitisation de la littérature ont pris des tournures spectaculaires et médiatiques avec le recours à la littérature comme outil de demande de justice et de dénonciation des violences sociales ou sexuelles. Dans le discours public, les écrivains contribuent à la circulation des problématiques transverses comme celle de l'anthropocène ou du genre, alors même que la culture est l'objet d'affrontements idéologiques nouveaux comme en témoignent les débats sur l'appropriation culturelle ou la woke culture : l'œuvre artistique est réinvestie d'une valeur éthique comme d'une portée politique. Alors même que la catégorie médiatrice d'intellectuel tend à disparaître, la frontière qui séparait les engagements privés de l'écrivain de son œuvre tend à céder, dans un double mouvement d'activation politique et de responsabilisation de l'art et de la littérature. Cette repolitisation a le double effet d'attribuer au travail artistique d'une force d'action et de l'exposer. L'écrivain devient directement imputable de son travail, non sans créer des effets de rétrospection troublants comme l'examen de conscience dont a fait l'objet en 2002 l'immunité complaisante dont a bénéficié Gabriel Matzneff. La peur, assez nouvelle, que des œuvres de fiction puissent blesser les sensibilités et véhiculer des contenus nocifs est donc un indice fort de ce réengagement dans des débats sociétaux : c'est, aux États-Unis, l'émergence dans les maisons d'édition de lecteurs destinées à prévenir toute offense, même involontaire, envers les communautés minoritaires, les sensitivity readers, en traquant les poncifs culturels rémanents avant publication, et les politiques universitaires visant à prévenir les étudiants par des trigger warnings de contenus potentiellement choquants dans des œuvres anciennes - tendance dont les virulentes polémiques entraînées par la mise au programme d'un poème de Chénier, L'Oaristys, supposé dépeindre de manière complaisante un viol, a montré qu'elle touchait désormais la France. La question est ici moins de savoir si ces procès prospectifs ou rétrospectifs relèvent d'une vigilance nécessaire ou de nouvelles formes de censure, que de constater qu'il est difficile d'attribuer au pharmakon littéraire une valeur thérapeutique sans en faire aussi un poison potentiel.

Quoi qu'il en soit, dans la fiction ou la non-fiction littéraire, aux larges grilles d'analyse héritées du marxisme se superposent des approches contextuelles et situées proposant des analyses fines des processus d'appropriation et des dominations, qui préfèrent l'écriture des terrains et les exemples des corps aux catégories abstraites des combats théoriques d'antan. Les politiques contemporaines de la littérature sont mues par des questionnements concrets sur les identités culturelles, performées par des œuvres conçues comme des dispositifs d'émancipation, incarnées par des corps qui avouent leurs blessures, agies sur le terrain par des écrivains qui vont écrire au plus près de populations vulnérables, théorisées par des essais littéraires situés dont King Kong théorie de Virginie Despentes a donné l'exemple. Elles se nourrissent d'hybridations avec la sociologie ou l'ethnologie et préfèrent souvent le savoir des sciences humaines aux matrices doctrinaires en rompant autant avec l'idéologie de l'autonomie esthétique qu'avec celle de l'engagement partisan. Pour toute une jeune génération, les modèles antérieurs, celui de l'écrivain engagé ou au contraire celui de l'écrivain dans sa tour d'ivoire, celui d'une littérature conçue comme force de progrès ou au contraire comme drame du langage, cèdent la place à une vision pragmatique des modes d'implication de l'écrivain et des pouvoirs de transformation de son discours, refusant tant l'humanisme abstrait que l'ère du soupçon. 
Demandes de justice environnementale, de réparation postcoloniale, de justice sociale, combat contre les invisibilisations historiques ou géographiques, sensibilisation aux violences du libéralisme et de la marchandisation du monde, attention aux causes des minorités, défense des migrants: les causes diverses défendues à la gauche de l'échiquier littéraire comme les inquiétudes identitaires de droite qui leur répondent, par exemple chez un Michel Houellebecq, ont en commun d'interpeller la démocratie contemporaine sans en contester les principes. S'il agit de corriger la démocratie, c'est pour souligner ses insuffisances en tant que démocratie. De fait, aussi bien les engagements des œuvres que la manière dont elles sont reçues par la société en font une contribution à l'exercice démocratique lui-même, consensus que relayent les discours politiques et institutionnels défendant le rôle démocratique de la culture. C'est sur les fondements de ce consensus qui exclut l'idée que la littérature soit indifférente à la démocratie comme l'hypothèse qu'elle puisse lui être contraire que l'on peut réfléchir : quelles sont les raisons invoquées à l'utilité démocratique de la littérature, idée réactivée à nouveaux frais la configuration sociopolitique du premier XXIe siècle?

En soi, le recours à la littérature dans le champ du débat public est aussi ancien que celui-ci la conception moderne de la littérature est contemporaine de la naissance de la démocratie libérale puisque l'émergence de l'idée d'autonomie politique des citoyens est parallèle à celle de l'autonomie du jugement esthétique - on pourrait s'amuser à dire qu'elles ont été rapportées en France d'Allemagne dans les valises de Benjamin Constant autour de 1800. Gisèle Sapiro en a largement fait l'histoire dans son essai Les écrivains et la politique en France. De l'Affaire Dreyfus à la guerre d'Algérie (Seuil, 2018) : recourant à la fiction comme outil critique de représentation des réalités sociales au XIXe, dans le roman réaliste ou le roman de formation, puis de point de vue individuels, situés et possiblement marginaux, au XXe siècle, le roman possède une fonction de "dévoilement " pour employer un terme sartrien, de dénaturalisation permettant d'analyser le champ social, et de conscientiser et de renverser les dominations symboliques. Cette dimension de dévoilement reste profondément active dans de nombreuses œuvres comme celles d'Édouard Louis, qui n'a pas peur de recourir à des modèles d'analyse simplifiés - on se souvient que dans Qui a tué mon père ?, l'écrivain rend directement responsable les politiques de la déchéance de son père: "Jacques Chirac et Xavier Bertrand détruisaient ses intestins ", " Nicolas Sarkozy et son complice Martin Hirsch lui broyaient le dos ", tandis que " Hollande, Valls et El Khomri l'ont asphyxié » et s'est adressé directement au président de la République par un Tweet « J'écris pour vous faire honte. J'écris pour donner des armes à celles et ceux qui vous combattent ".

La dimension de contestation et la puissance de radicalité de la littérature s'expriment traditionnellement dans des formes réalistes, qu'elles soient celles d'une description romanesque large d'un territoire ou d'un groupe social. De Michel Houellebecq, d'Éric Vuillard, de Leïla Slimani, ou encore de Nicolas Mathieu, pour prendre quelques prix Goncourt de ces dernières années, le roman projette sa réflexion sur les inégalités sociales contemporaines. À côté des nombreux romanciers qui mettent en voix les invisibles et les laissés pour compte, SDF, petits employés, banlieusards, en proposant une observation fine, une description dense analogue à celle promue par l'anthropologie et une attention aux pratiques ordinaires, le roman romanesque plus traditionnel ne cesse de dramatiser pour l'analyser un monde économique en tension : de Karine Tuil à Éric Reinhardt, le regard est porté sur les mécaniques du travail à l'heure managériale, les crises environnementales, les vulnérabilités territoriales et les dépendances produites par nos économies mondialisées. Le roman de l'ère démocratique avait servi aux $\mathrm{XIX}^{\mathrm{e}}$ et $\mathrm{XX}$ siècles les combats d'émancipation ; 
lorsqu'elle est privée de doctrine, ne mobilisant pas de grandes solutions, la littérature contemporaine poursuit néanmoins cette fonction d'alerte par une scénarisation des nouveaux rapports de force et nouvelles luttes de classes induites par le libéralisme. À une heure où le Grand soir n'est plus qu'une rêverie nostalgique et où les démocraties occidentales se sentent à nouveau menacées dans leur hégémonie, cette critique du système est intégrée au débat démocratique et la puissance de contestation des écrivains est entérinée par les institutions et même l'école, dans un jeu de rôle bien codifié.

À côté d'un paradigme cognitif qui fait de la littérature un mode d'accès à la vérité profonde des rapports et des structures dans des narrations réalistes, on a pu souligner la fonction de critique portée par le langage littéraire lui-même, et l'on a pu revendiquer l'utilité démocratique de sa fonction d'écart et de dénaturalisation des discours. Par l'écriture, la littérature viendrait ainsi mettre en scène et déconstruire le storytelling politique et les discours médiatiques, dans des jeux littéraires débridés pratiqués par exemple par des écrivaines situées aussi fortement à gauche que Nathalie Quintane, Emmanuelle Pireyre ou encore la jeune Sandra Lucbert. Car de manière tout à fait intéressante, le grand formalisme (pensons à Duras) ou le postmodernisme français (pensons à Perec) a revendiqué à la fois l'expérimentation d'un langage en rupture avec les "mots de la tribu " (Mallarmé) et l'ambition de transformation politique. Cette postulation, en apparence contradictoire, se trouvera théorisée chez un Barthes qui promouvra une éthique de la forme et une politique de l'expérimentation littéraire et incarnée par des écrivains comme Pierre Guyotat ou Philippe Sollers. Pour ceux-ci, il n'y aura pas d'écart entre la nécessité d'accessibilité démocratique et une production esthétique n'ayant pas peur de la difficulté voire de l'hermétisme: la vraie révolution se jouerait encore dans l'écriture, hypothèse que l'on retrouve encore dans le discours de bien des écrivains d'aujourd'hui. C'est à ce titre là que l'on a pu revendiquer sans paradoxe que le poétique serait d'ordre politique, comme le fait, parmi d'autres, le poète Jean-Michel Espitallier, définissant la poésie comme un genre " insoumis ».

L'autonomisation au XIX ${ }^{\mathrm{e}}$ siècle de la littérature des enjeux moraux et sociaux du monde commun, renforcé par la marginalisation sociale des écrivains dans l'art pour l'art au milieu du XIX siècle bien décrite par Bourdieu, et la transformation de l'exclusion sociale en une royauté symbolique dans le monde séparé du langage, a pu conduire les écrivains artistes à se couper de la cité. L'écrivain n'y participerait que par une politique de l'écart et du retrait, par des provocations extérieures. Mais le surinvestissement dans le langage comme monde autosuffisant a pu être revendiqué politiquement: Jacques Rancière avance ainsi le rôle essentiel de la proposition esthétique, qui par sa liberté rappellerait l'absence de prédétermination dans le découpage du sensible et souligne que la littérature confronte des formules de description de la réalité toutes considérées comme égales et pourtant contradictoires, au bénéfice de la démocratie. Le travail de la forme permet d'inclure le réel dans la représentation littéraire en dehors des hiérarchies opposant des objets ou des personnes dignes et d'autres, moins dignes d'une mimésis. Loin d'être une valeur de droite liée à la tradition et à la proximité des écrivains avec le pouvoir, l'investissement dans le style est alors, autant que l'attention aux dominés, une caractéristique de la gauche littéraire.

Dévoiler, subvertir, promouvoir des contre-discours comme autant de contre-pouvoirs : les théories cognitives ou linguistiques de l'action politique de la littérature héritées du passé persistent dans le champ contemporain. Mais celui-ci voit également l'émergence d'autres formes de promotion des vertus démocratiques de la littérature, qui insistent moins sur la fonction d'écart et de critique que sur celle d'intégration et de lien. L'autonomie de la littérature était une valeur d'émancipation face à des pouvoirs politiques autoritaires, des 
sociétés holistes, des organisations du travail verticales et des systèmes familiaux contraignants, et elle a pu l'être encore récemment dans des pays soumis à des régimes autoritaires. Mais dans les sociétés contemporaines libérales individualisantes aux systèmes de sens et de valeurs souvent déstructurées, mettant en avant la fluidité et la circulation des identités, et exposant chacun aux risques de l'isolement et de la déliaison, la demande politique qui s'exerce à l'égard de la littérature (et à l'égard de l'art en général) est au contraire une demande de relation. Cette inversion me semble cruciale pour comprendre le devenir de la littérature contemporaine, ses discours d'intervention et de réparation, comme les thèmes de l'attachement et de la communauté qui en parcourent les discours. Elle conditionne un tournant pragmatique de la littérature, qui fait de celle-ci autant une expérience qu'une vision du monde : la littérature devient de plus en plus au XXI siècle un outil d'émancipation d'abord individuelle, puis, par la viralité de la lecture, sociale. Elle concourrait à la démocratie en promouvant des pratiques de connaissance intime et d'émancipation individuelle autant qu'en délivrant des images globales ou des discours généraux. C'est ainsi dans les romans de trauma et d'émancipation, de Christine Angot à Vanessa Springora, ou dans les grands récits intimes de notre temps, de l'autobiographie collective Annie Ernaux aux introspections empathiques d'Emanuel Carrère, dans lesquelles le lecteur est invité à la réflexion intime sur la situation du sujet et sur ses connexions au monde, que l'on peut peut-être trouver les plus fortes "micropolitiques » de la littérature contemporaine, pour reprendre le terme de Félix Guattari. À défaut de discours généraux, ces écritures du sujet manifestent une attention à soi tout en promouvant une empathie qui déjoue les rapports de force institués et interdisent l'indifférence, travers possible de l'individualisme libéral. La circulation des points de vue et le dialogue de perspectives situées compensent l'absence de grands récits et la dislocation des systèmes de sens collectifs. "Sans récit et sans image pour relier les sous-groupes, le monde social est fragmenté par l'ignorance et le sentiment d'étrangeté ", explique Alice Zéniter dans un essai récent justifiant le besoin de récits (Je suis une fille sans histoire, L'Arche, 2021), appel qui contraste avec la défiance contre la fiction et même contre la narration en régime " médiocratique » saturé de récits officiels, pour emprunter les formules d'Yves Citton.

Le besoin de littérature, de fiction ou de non-fiction, comme d'un outil de connaissance de soi et de développement personnel submerge les librairies et les discours médiatiques, alors que le numérique et les réseaux sociaux facilitent concrètement la démocratisation de l'écriture, qui poursuit et prolonge la démocratisation de la lecture qui avait caractérisé l'ère moderne. Mais ce mouvement est indissociable de l'importance nouvelle des pratiques sociales accompagnant la littérature, résidences d'écrivains, festivals, clubs de lecture, ou atelier d'écriture à un moment où la reconnaissance de l'importance " citoyenne " de littérature et de ses intermédiaires, libraires et éditeurs, semble s'être imposée profondément dans les démocraties. La littérature est exposée dans les rames de métro ou sur les abribus, elle est financée par les pouvoirs publics comme un outil facilitant le lien social et se trouve promue comme instrument de constitution d'un monde commun. Certaines pratiques amateures d'écriture collaborative (pensons par exemple aux pratiques collaboratives d'écriture sur des plates-formes comme Wattpad ou Fanfiction.net) expérimentent des modalités originales d'organisation démocratique horizontale tandis que les écrivains théorisent les manières nouvelles de dire "nous » (voir par exemple Nous de Tristan Garcia, Grasset, 2016) ou, très concrètement, investissent des zones à défendre écologiquement menacées (voir sur ce sujet le travail du collectif ZoneZadir). Le travail de recueil de terrain et de réécriture des paroles ouvrières pour y inscrire la polyphonie inquiète du corps social et produire une solidarité énonciative de François Bon dans Daewoo a été une expérience littéraire autant qu'humaine 
marquante de l'histoire littéraire récente. Dans le théâtre contemporain, les expériences "démocratiques" sont pléthoriques et anciennes: le théâtre ne cesse de proposer des formes participatives nouvelles depuis les années 1960, du travail Joël Pommerat à celui de Rimini Protokoll. À mi-chemin entre le forum citoyen et la performance, la production de procès fictionnels ou de "parlements » (parlement des choses de Bruno Latour, parlement de la Loire de Camille Toledo, etc.) illustre d'autres rapprochements possibles du théâtre et de la réflexion politique.

Dans les discours sur l'écriture, le projet de réunion d'une communauté à travers les résonnances et les liens faibles de la littérature corrige l'inévitable retrait de l'écrivain. L'empowerment individuel du lecteur est conçu comme le préalable nécessaire à la constitution de communautés nouvelles, locales et situées, où peut se réunir " le peuple qui manque " pour reprendre l'expression célèbre de Deleuze, en réparant la dislocation de son identité, la destruction de son rapport au travail et la perte de ses ancrages locaux causées par des décennies de crise économique et de délitement des discours de référence. La critique prend acte de ces nouvelles ambitions politiques de la littérature. Issue des travaux de Martha Nussbaum, la promotion néo-humaniste de l'écriture comme encouragement au «vivre ensemble " à travers l'éducation morale sensible de chacun permise par le roman, dispositif empathique par lequel nous apprendrions la souffrance d'autrui et la reconnaîtrions, a connu en France une réception enthousiaste. Moins connue, mais influente, Louise Rosenblatt, fait de la littérature un réservoir d'expériences humaines possibles susceptibles de favoriser l'ouverture sur le monde, la rencontre avec l'autre et l'émancipation de l'individu. En France, la manière dont Nelly Wolf analyse le roman moderne comme " une mimésis du nouveau contrat social, celui qui fonde la démocratie moderne " et une sorte de simulation par la lecture des postures et des réflexes démocratiques contribue à proposer des fondements théoriques à cette conception de la littérature comme initiation au vivre ensemble. Que l'on voie dans la littérature une simulation distanciée ou un transfert empathique direct, la fabrication de consensus ou la catharsis de conflictualités, essayistes et théoriciens redeviennent attentifs à l'entraînement des vertus démocratiques par la littérature, exercice de découverte et de sortie de soi favorisant le sens de l'égalité et du pluralisme.

II serait bien vain de circonscrire par un paradigme unique les politiques contemporaines de la littérature. Analyses ethnologiques ou sociologiques des conditions, discours d'autosaisie des identités et d'émancipation, mise en scène épique des dominations, promotion de la furtivité ou l'invisibilité comme mode de résistance et déconstruction anti-autoritaire des discours, mise en circulation d'utopies ou au contraire de dystopies, ont pourtant en commun de promettre une effectivité nouvelle : renonçant à tout paternalisme et à tout discours de surplomb, réticentes à imposer l'universalité de catégories transverses et à verrouiller des modes de représentations, attentives à l'illégitimité potentielle des prises de paroles, les politiques littéraires d'aujourd'hui défendent néanmoins toutes le pouvoir concret de l'écriture et de la lecture individuelle comme exercice effectif d'une vie démocratique relationnelle fondée sur l'attention concrète à la pluralité des formes de vie et à la justesse de leur énonciation. Que ce consensus original et pragmatique en faveur de la démocratie puisse être utile à des sociétés menacées par la montée des démagogies et des discours autoritaires, est le vœu le plus cher de l'auteur de cet article. 